\title{
Phoretic dynamics of colloids in a phase separating critical liquid mixture
}

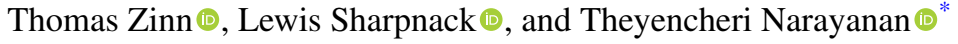 \\ ESRF - The European Synchrotron, 38043 Grenoble, France
}

(Received 26 February 2020; accepted 8 July 2020; published 31 July 2020)

\begin{abstract}
Different phoretic effects have been widely exploited for designing self-propelled colloidal systems, but the underlying wave vector dependent dynamics has been little investigated. In this work, the out-of-equilibrium dynamics of colloids in near-critical liquid mixtures undergoing spinodal phase separation was probed by $\mathrm{x}$-ray photon correlation spectroscopy (XPCS). The emergent dynamics of charge stabilized silica and silica-nickel Janus particles upon a temperature jump into the two-phase region of the solvent mixture displayed similar features at the initial stage. In both systems, the phoretic dynamics is dominated by velocity fluctuations induced by the solvent concentration fluctuations and the hydrodynamic flow during the coarsening process. Furthermore, relaxation rates of the diffusive part of the dynamics manifested an anomalous wave vector dependence akin to the superdiffusive behavior with an effective diffusion coefficient significantly larger than the Brownian limit. For smaller temperature jumps, velocity fluctuations exhibited a broader distribution with silica and Janus colloids showing qualitatively different behavior. The velocity fluctuations decayed with time and the dynamics reverted to diffusive behavior upon completion of the phase separation. Presented results illustrate the ability to probe faster collective dynamics pertinent to active colloids using multispeckle XPCS.
\end{abstract}

DOI: 10.1103/PhysRevResearch.2.033177

\section{INTRODUCTION}

Over the last two decades, phoretic motions of particles have received renewed interest from the perspective of designing active colloids, which consume energy from the surrounding medium and perform mechanical motion [1-3]. Self-phoretic motions can be induced by a variety of ways, but the diffusiophoresis that involves the gradient of concentration is one of the most widely used mechanism for designing active colloids [2,3]. Such self-propelled colloids are simple manifestations of active matter that has been the subject of burgeoning research over the past several decades [4-6]. Apart from the fascinating observations of their collective behavior, understanding the interaction and dynamics underlying the phoretic effects are important from both fundamental and practical points of view [2,3,7]. The active motions of such particles can be easily tracked in two dimensions (2D) by optical microscopy and therefore they have been widely investigated $[5,6]$.

An elegant approach for realizing self-phoretic motion is by selective heating of one of the faces of Janus particles suspended in a critical binary liquid mixture [8]. The local demixing of the solvent in the vicinity of the heated face results in a gradient of concentration, a condition analogous to the diffusiophoresis. The swimming direction of the individual particles is determined by the preference of the heated face

\footnotetext{
*Corresponding author: narayan@esrf.fr

Published by the American Physical Society under the terms of the Creative Commons Attribution 4.0 International license. Further distribution of this work must maintain attribution to the author(s) and the published article's title, journal citation, and DOI.
}

for a given liquid species [8]. This has provided a convenient method for investigating the emergent collective behavior of active colloids [8,9]. Colloids dispersed in binary liquid mixtures exhibit many fascinating features near the coexisting region of the solvent, which have been the subject of numerous investigations in the past [10-12]. Near the coexistence temperature $\left(T_{\mathrm{cx}}\right)$, one of the liquid species preferentially adsorbs on the surface of colloids $[10,13]$. Theoretical investigations of the propulsion mechanism of self-heated active Janus particles suggested different origins such as wetting and associated surface forces [14], and chemical potential gradients at the diffuse interface [15] upon local demixing of the solvent. The diffusiophoresis picture is insufficient to explain all the observed features and more recently it has been shown that gradient of mechanical stresses in the adsorbed layer, analogous to the Marangoni effect is likely the propulsion mechanism [16].

An important aspect of colloids in binary liquid mixtures is that the interaction between particles can be tuned by varying the temperature $(T)$ in the proximity of the consolute point $[12,13]$. Near $T_{\mathrm{cx}}$ of the liquid mixture, charge stabilized colloids turn attractive and aggregate at a well-defined temperature $\left(T_{a}\right)$. This aggregation process is thermally reversible and it is closely related to the preferential adsorption of one of the liquid species on to the colloids [13]. In addition, a reentrant stability of the suspension can be observed in the coexisting region with all particles redispersing in the preferred phase [10]. All these features can be exploited to modify the interactions and dynamics of colloids [17]. A wider range of tunability is achieved by using Janus colloids having faces with different wetting properties [18-20].

In the case of active colloids in binary liquid mixtures, a better understanding of the change in interactions and dynamics upon the phase separation of the solvent is desired, 
prior to assigning different observations to the physics of active matter. In particular, the dynamics of colloids when the solvent is undergoing spinodal phase separation has not been investigated in detail presumably due to the high turbidity of the system. In this work, the evolution of the microstructure and dynamics of colloids were probed simultaneously by ultrasmall-angle x-ray scattering (USAXS) and multispeckle ultrasmall-angle x-ray photon correlation spectroscopy (USAXPCS), respectively. Here, the term phoretic dynamics implies the enhanced dynamics of colloids induced by concentration fluctuations upon the phase separation of the solvent mixture and subsequent hydrodynamic flow during the coarsening process. The USA-XPCS provides access to the wave vector dependent ensemble averaged dynamics, which can be decomposed to advective and diffusive contributions [21]. Especially, the deviations from the mean velocity or velocity fluctuations of the particles can be deduced with high statistical accuracy. For active Brownian particles, their motion remains ballistic over a limited time defined by the rotational diffusion, beyond which their behavior becomes stochastic [22]. Therefore the ensemble averaged dynamic information in three dimensions (3D) derived from scattering experiments is important and complements the results obtained by optical microscopy in 2D.

As model colloids, spherical silica and silica-nickel Janus particles (SP and JP, respectively) were used. These particles were dispersed in near-critical quasi-binary solvent mixtures composed of 3-methylpyridine (3MP), water (W) and heavy water $(\mathrm{HW})$, which has a closed-loop phase diagram with a lower critical solution temperature, $T_{c}$. The behavior of colloidal particles in this mixture is similar to that in the more well-studied 2,6-lutidine and water mixture [23]. However, there is an additional tunability of $T_{c}$ by varying the $\mathrm{W}$ to $\mathrm{HW}$ ratio. At the critical concentration $\left(C_{c}\right)$, the solvent mixture phase separates predominately via spinodal decomposition [24]. Thanks to the very low $x$-ray contrast of the solvent mixture, $\mathrm{x}$-ray scattering is dominated by colloids that enabled the investigation of interparticle interactions and dynamics in the turbid medium when the solvent mixture is undergoing spinodal decomposition. The phase separation in this case was induced by a temperature jump ( $\mathrm{T}$ jump), which is not exactly the same condition as that involved when particles are heated locally by laser illumination [8]. Nevertheless, the underlying processes of spinodal phase separation and preferential adsorption are similar in both cases.

The spinodal decomposition in this type of binary liquid mixtures has been extensively studied [25-28] and reasonably well described in terms of different models [24,29-31]. For the T jumps used in this study, the solvent mixture immediately reaches the fast growth regime dominated by surface tension and gravity induced hydrodynamic flows. In particular, the crossover range from capillary (interfacial tension) driven to gravity driven flow when the domain sizes reach tens of micrometers $[27,29]$ is the relevant condition for this work. During the phase separation of the solvent, particle dynamics changed from purely Brownian to ballistic behavior, with the advective part dominated by particle velocity fluctuations induced by solvent concentration fluctuations and the hydrodynamic flow. Decomposition of the measured intensity-intensity autocorrelation function, $g_{2}(q, t)$, enabled

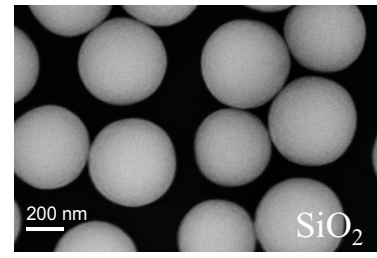

(a)

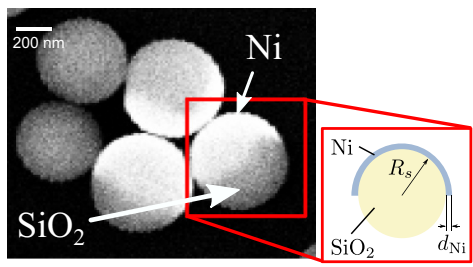

(b)
FIG. 1. SEM micrographs of (a) silica and (b) silica-nickel Janus colloids. The sketch schematically illustrates a hemispherical nickel cap on the silica sphere.

the extraction of an effective diffusion coefficient, the mean velocity fluctuations and the average flow velocity of particles in the scattering volume.

Previous studies on the same system mainly focused on off-critical concentrations and isotropic dynamics, when the solvent phase separation occurred predominantly via nucleation and growth $[17,20]$. Recent advances in x-ray detector technology (e.g., the Eiger pixel-array detector) in terms of higher spatial and temporal resolution [32], enabled the investigation of shorter time range and perform a direction dependent analysis of the particle dynamics. The high frame rate capability of the Eiger-500k detector together with the high photon flux permitted the measurement of ensemble averaged $g_{2}(q, t)$ functions with a high signal-to-noise ratio in the sub-millisecond time range. This allowed the disentanglement of the emergent particle dynamics and in particular more quantitative extraction of parameters. In addition, wider range of $\mathrm{T}$ jumps were employed to explore the evolution of particle dynamics as a function of distance from $T_{c}$.

\section{EXPERIMENTAL}

\section{A. Sample preparation}

Spherical colloidal silica particles (SP) with a mean radius $\left(R_{S}\right)$ of $230 \mathrm{~nm}$ and a polydispersity $(P D) \simeq 0.05$ (SchulzZimm distribution) were synthesized by the Stöber method [17]. Silica-nickel Janus particles (JP) were prepared by sputtering nickel onto a monolayer of silica particles formed on silicon substrates by solvent (ethanol) evaporation [20]. The resulting films were extracted by sonication and redispersed in water. A more detailed description of the JP preparation can be found in a previous publication [20]. The particles were characterized by electron microscopy and USAXS. Figures 1(a) and 1(b) show scanning electron microscope (SEM, ZEISS LEO 1530) images for SP and JP, respectively. The nickel coating on the silica particles is visible as a brighter region in Fig. 1(b) as schematically shown in the sketch. In addition, the characteristic line of $\mathrm{Ni}$ was visible in the emission spectrum. The final particles had $R_{S} \simeq 200 \mathrm{~nm}$ and nickel layer thickness $\left(d_{\mathrm{Ni}}\right) \simeq 40 \mathrm{~nm}$, as determined by USAXS modeling. The thickness of nickel layer is significantly larger than the particles used in the previous work [20]. JP and SP were dispersed in the solvent mixture composed of 3-methylpyridine (3MP), water (W), and heavy water (HW) (JP just before the $\mathrm{x}$-ray measurements to minimize their slow aggregation). The weight fraction of $3 \mathrm{MP}\left(C_{3 \mathrm{MP}}\right)$ was adjusted to the critical value $\left(C_{c}\right)$ of 0.3 for a W to $\mathrm{HW}$ ratio of $1: 4$ by weight 


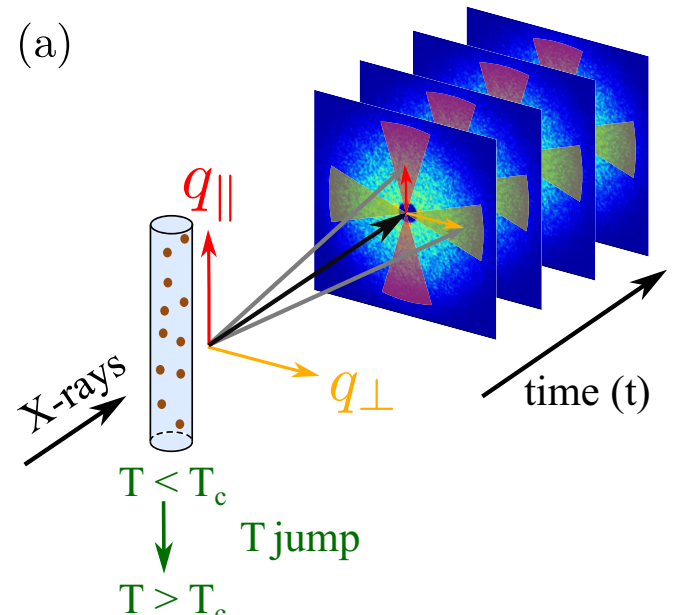

(b)

Silica

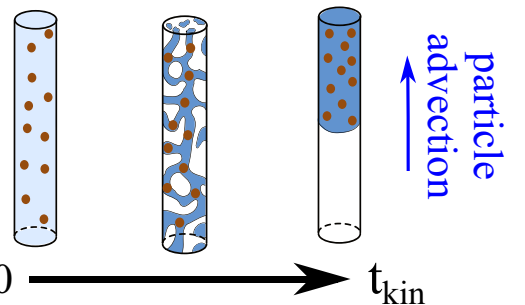

(c)

Janus

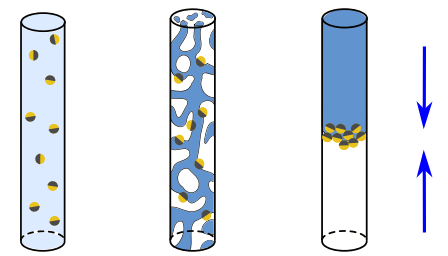

FIG. 2. (a) Illustration of the XPCS experimental scheme following a $\mathrm{T}$ jump. The direction dependent analysis is indicated by the shaded arcs in the 2D scattering pattern: parallel and perpendicular to the gravity, $q_{\|}$(vertical), and $q_{\perp}$ (horizontal), respectively. Schematic representation of the systems (b) SP and (c) JP before and after the T jump, and the distribution of particles. The kinetic time $\left(t_{\text {kin }}\right)$ starts from 0 when the sample reaches the set temperature.

to obtain $T_{c}$ around $43{ }^{\circ} \mathrm{C}$. Suspensions were transferred to flat-walled capillary cells of width $2 \mathrm{~mm}$ and inner path length $0.5 \mathrm{~mm}$ (wall thickness $0.1 \mathrm{~mm}$ ), and flame sealed.

Prior to XPCS experiments, slow temperature scans were performed in order to determine $T_{a}$ and $T_{c}$ precisely. The stability of the suspension was determined from the measured USAXS profile at each scan point. For SP, $T_{a}$ and $T_{c}$ were at about $41.5^{\circ} \mathrm{C}$ and $43.6^{\circ} \mathrm{C}$, respectively. Note that values of $T_{a}$ and $T_{c}$ are also sensitive to any remnant impurities in the suspension. Unlike SP system, JP samples do not show a sharp thermally reversible aggregation but a slow irreversible aggregation which is accelerated in the vicinity of the coexistence region. The $T_{c}$ of JP suspensions was about $44.8^{\circ} \mathrm{C}$ and this difference in $T_{c}$ of about $1 \mathrm{~K}$ could be due to variations in the $\mathrm{W}$ to $\mathrm{HW}$ ratio [23].

Figure 2 schematically depicts the experimental setup and changes to the sample upon a $\mathrm{T}$ jump. Fast and reproducible T jumps were performed using a Mettler Toledo heating stage (FP85) at a heating rate of $0.33 \mathrm{~K} / \mathrm{s}$. T jumps were carried out from the one-phase region into the two-phase region, typically from $40{ }^{\circ} \mathrm{C}$ to the desired temperature. The difference between the final temperature and $T_{c}$ is denoted as $\Delta T$ and it is the control parameter for the phase separation dynamics of the solvent mixture. For very small values of $\Delta T \simeq 0.05 \mathrm{~K}$, a temperature controlled oven with $\mathrm{mK}$ stability was employed. However, in that case, the heating rate was approximately 20 times slower $(0.016 \mathrm{~K} / \mathrm{s})$.

\section{B. X-ray scattering setup}

All experiments were carried out at ID02 beamline of the European Synchrotron Radiation Facility (ESRF) in Grenoble, France [33]. The incident X-ray energy was $12.46 \mathrm{keV}$, corresponding to a wavelength, $\lambda=0.995 \AA$. The sample-to-detector distance was set to $30.7 \mathrm{~m}$ for accessing the $q$ scales pertinent to the two systems investigated. The coherent beam was selected by a pair of slits of aperture $30 \mu \mathrm{m}$ (vertical) $\times 20 \mu \mathrm{m}$ (horizontal), which provided a photon flux of $5 \times 10^{10} \mathrm{ph} / \mathrm{s}$ at the sample position. With these dimensions, the beam was roughly elliptical on the sample and together with the path length of $0.5 \mathrm{~mm}$ defined the scattering volume. 2D speckle patterns as illustrated in Fig. 2(a) were acquired using an Eiger-500k detector with a pixel size of $75 \mu \mathrm{m}$ in 8-bit mode [32]. The resulting speckle contrast was better than $30 \%$ at $30.7 \mathrm{~m}$ sample-to-detector distance. Further details of the experiment setup for XPCS is described elsewhere [32]. Depending on the dynamics, the acquisition rate varied from 2 to $10 \mathrm{kHz}$ and typically 2000 to 5000 frames were acquired in order to capture the full decay of $g_{2}(q, t)$. The static scattering profiles were obtained by the azimuthal average of the 2D USAXS patterns after applying different corrections and normalization to an absolute intensity scale [33]. The normalized background subtracted USAXS intensity is denoted by $I(q)$.

\section{XPCS principle and data reduction}

In a coherent scattering experiment, the measured speckles fluctuate due to the spatio-temporal rearrangement of the scattering objects in the medium. The intensity fluctuations can be quantified by the homodyne intensity-intensity autocorrelation function [34],

$$
g_{2}(\mathbf{q}, t)=\frac{\left\langle I\left(\mathbf{q}, t_{0}\right) I\left(\mathbf{q}, t_{0}+t\right)\right\rangle}{\left\langle I\left(\mathbf{q}, t_{0}\right)\right\rangle^{2}}
$$

with $I(\mathbf{q}, t)$ being the scattered intensity measured at a scattering vector, $\mathbf{q}$, lag time, $t$, and $\langle\cdot\rangle$ denotes the time average over the duration of the measurement. The magnitude of $\mathbf{q}$ is given by $q=4 \pi / \lambda \sin (\theta / 2)$ with $\theta$ the scattering angle. $g_{2}(q, t)$ is related to the intermediate scattering function or the first order field-field autocorrelation function, $g_{1}(q, t)$, via the Siegert relation [34]:

$$
g_{2}(q, t)=1+\beta\left|g_{1}(q, t)\right|^{2}
$$

where $\beta$ is the speckle contrast that depends on the coherence properties of the incident $\mathrm{x}$-ray beam and the resolution of the scattering setup. The dynamic information of the systems is contained in $g_{1}(q, t)$. For the simple case of noninteracting diffusive Brownian particles, $g_{1}(q, t)$ decays exponentially:

$$
g_{1}(q, t)=\exp [-\Gamma(q) t]
$$


where the relaxation rate, $\Gamma(q)=D_{0} q^{2}$ and $D_{0}$ is the StokesEinstein diffusion coefficient given by $D_{0}=k_{B} T /\left(6 \pi \eta R_{H}\right)$ with $k_{B}$ the Boltzmann constant, $\eta$ the viscosity of the solvent and $R_{H}$ the hydrodynamic radius of particles [34].

However, when particles are in a directed motion, their net displacement is contributed by both advection and diffusion [35],

$$
\mathbf{r}_{j}(t)-\mathbf{r}_{j}\left(t_{0}\right)=\Delta \mathbf{r}_{j, D}(t)+\mathbf{v} t+\Delta \mathbf{v}_{j} t,
$$

where $\Delta \mathbf{r}_{j, D}$ corresponds to the change in position of the particle $j$ due to Brownian motion, $\mathbf{v}$ is the mean velocity of all particles in the scattering volume and $\Delta \mathbf{v}_{j}$ is the relative velocity of the particle $j$ from the mean velocity. The resulting $\left|g_{1}(q, t)\right|^{2}$ for all particles in the scattering volume can be factorized as follows [35-37]:

$$
\left|g_{1}(q, t)\right|^{2}=\left|g_{1, D}(q, t)\right|^{2}\left|g_{1, T}(q, t)\right|^{2}\left|g_{1, A}(q, t)\right|^{2}
$$

In Eq. (5), the first term describes the diffusion of particles similar to that in Eq. (3), the second term accounts the decorrelation effect when a particle moves out completely from the scattering volume and the third term is the leading contribution due to advection. In the homodyne detection scheme, the intensity fluctuations are modulated by the difference in Doppler shifts caused by all particle pairs in the scattering volume and it is given by the Fourier cosine transform of the probability distribution of velocity differences (velocity fluctuations, $\delta v)[35,38]$. For a Gaussian distribution of velocity fluctuations and a Gaussian beam profile with width $w$, Eq. (5) leads to $[35,39]$

$$
\begin{aligned}
g_{1}(q, t)= & \exp \left(-D_{\text {eff }} q^{2} t\right) \exp \left[-\left(t / t_{T}\right)^{2}\right] \\
& \times \exp \left(-q^{2} \delta v^{2} t^{2} / 2\right),
\end{aligned}
$$

where $D_{\text {eff }}$ is an effective diffusion coefficient which may deviate from $D_{0}$ during the advection and $t_{T}$ is the transit time given by $t_{T}=w / v$. Note that the mean velocity, $v$ is roughly the same throughout the bulk of the sample, whereas $\delta v$ is a relative quantity that scales with the size of the scattering volume (path length).

In principle, all these three terms can be decomposed from their differences in the $q$ dependence. However, for the experimental conditions used here (beam size and path length) and $v$ values involved, the transit term was found to be not significant, i.e., $\left|g_{1, T}(q, t)\right|^{2} \approx 1$ [37] and $\delta v$ term dominated the decay of $g_{1}(q, t)$. Furthermore, measured $g_{2}(q, t)$ functions showed characteristic feature of a constant velocity difference [40]. For a uniform distribution of $\delta v$ around the mean velocity $v,\left|g_{1, A}(q, t)\right|$ can be expressed analytically as [41]

$$
\left|g_{1, A}(q, t)\right|^{2}=\left[1-\alpha+\alpha \frac{\sin (q \delta v t)}{q \delta v t}\right]^{2}
$$

where the parameter $\alpha$ denotes the fraction of advecting particles with velocities deviating from $v$. Substituting Eq. (7) in Eq. (5) and neglecting $\left|g_{1, T}(q, t)\right|^{2}$ term,

$$
\begin{aligned}
g_{2}(q, t)= & 1+\beta\left[1-\alpha+\alpha \frac{\sin (q \delta v t)}{q \delta v t}\right]^{2} \\
& \times \exp \left[-2 \Gamma_{\mathrm{eff}}(q) t\right],
\end{aligned}
$$

where $\Gamma_{\text {eff }}(q)$ is an effective relaxation rate that may deviate from pure Brownian behavior during the advection. In analogy with Eq. (7), when only $\alpha$ fraction of advecting particles deviate from $v$ and Gaussian distributed,

$$
\begin{aligned}
g_{2}(q, t)= & 1+\beta\left[1-\alpha+\alpha \exp \left(-q^{2} \delta v^{2} t^{2} / 2\right)\right]^{2} \\
& \times \exp \left[-2 \Gamma_{\text {eff }}(q) t\right] .
\end{aligned}
$$

From the measured speckle patterns, $g_{2}(q, t)$ functions were calculated using the python program (PyXPCS) developed at the ESRF. Each correlation function $g_{2}(q, t)$ was calculated pixel-by-pixel for different $q$ values and then azimuthally averaged over all speckles corresponding to a given $q$ bin $\left(\Delta q=5 \times 10^{-4} \mathrm{~nm}^{-1}\right)$ in order to obtain the time and ensemble averaged $g_{2}(q, t)$.

For anisotropic dynamics, the ensemble averaged $g_{2}(q, t)$ functions were calculated by taking only an azimuthal sector of $\pm 15^{\circ}$ along the vertical and horizontal directions as indicated in Fig. 2(a). The $g_{2}(q, t)$ is not sensitive to the reversal of the direction up or down and left or right. As a result the averaging for the vertical and horizontal directions were performed using both upper and lower sectors, and left and right sectors, respectively.

\section{RESULTS AND DISCUSSION}

\section{A. Phase behavior}

The phase behavior of charge stabilized SP in this quasibinary liquid mixture is well-established [10]. 3MP preferentially adsorbs onto silica particles near $T_{a}$ leading to a thermoreversible aggregation. In the two-phase region, particles become stable again (reentrant stability) and migrate completely to the 3MP-rich phase. A schematic phase diagram for SP system is shown in Ref. [42]. In addition, the dynamics of SP in off-critical mixtures were reported in an earlier publication [17].

The phase behavior of silica-nickel JP is significantly different as schematically depicted in Fig. 3. Due to the limited stability of the suspension (resulting from the low surface charge on the cap and large Hamaker constant of nickel, which make the faces weakly attractive), the particles irreversibly aggregate over a time scale of several days at room temperature [20]. This aggregation is accelerated near the phase boundary as schematically indicated by the gray region in Fig. 3. The photograph in Fig. 3 illustrates the macroscopic observation after a $\mathrm{T}$ jump into the two-phase region for a $\Delta T \approx 4.2 \mathrm{~K}$. Immediately upon phase separation, particles migrate to the liquid-liquid interface. From the macroscopic spreading behavior observed with silica and nickel particleladen surfaces, it was found that 3MP wets completely both silica and nickel, with slight preference for the latter, whereas water wets only silica. Therefore, it can be inferred that nickel prefers $3 \mathrm{MP}$ and silica switches the preference to water, as previously suggested [20]. With increasing JP concentration, it becomes unsustainable for the interface to accumulate all particles since the interfacial tension (that vanishes at $T_{c}$ ) is very small [11]. As a result, particles cluster into different configurations [18] nickel-nickel and silica-silica and distribute into the preferred phase or collapse to the bottom of the capillary, as indicated in Fig. 3. The anisotropic Janus 


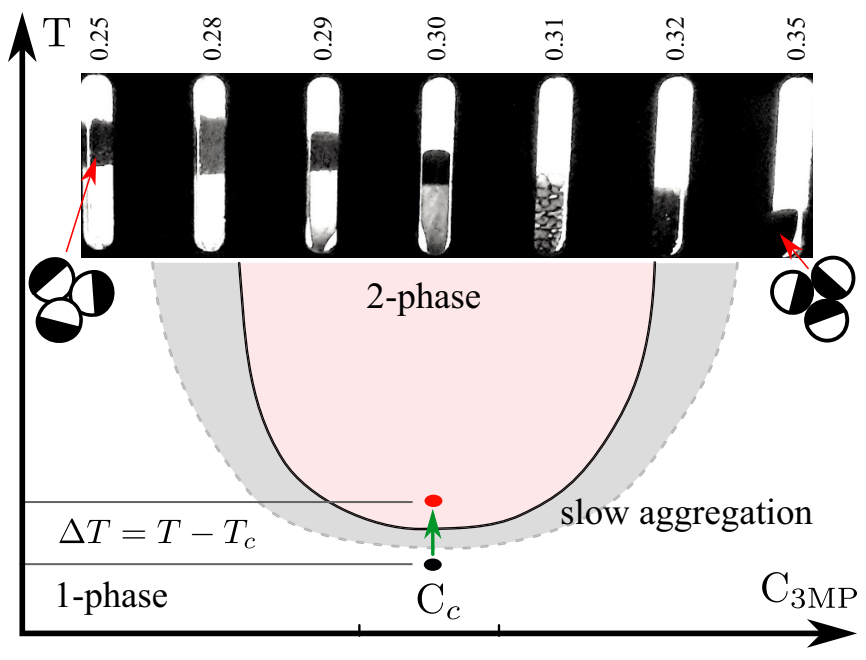

FIG. 3. Schematic phase diagram of silica-nickel Janus particles in the quasi-binary mixture of 3-methylpyridine (3MP), water (W) and heavy water (HW). Photograph shows the macroscopic final phases for a $\Delta T \approx 4.2 \mathrm{~K}$ with clusters migrating to preferred phases. In $C_{3 \mathrm{MP}}=0.31$ sample, particles can be seen at the interface of large domains. Within the scheme of aggregates (insets), silica and nickel are represented by the white and black hemispheres, respectively.

interaction may favor the formation of clusters with attractive sides (face-to-face) close to each other and repulsive sides (back-to-back) staying apart [43], with the preferred face of solvent exposed.

\section{B. Static characterization}

Prior to the XPCS measurements all samples were characterized by static USAXS measurements in the same capillary cell both below and above $T_{c}$. Figure 4 displays the normalized

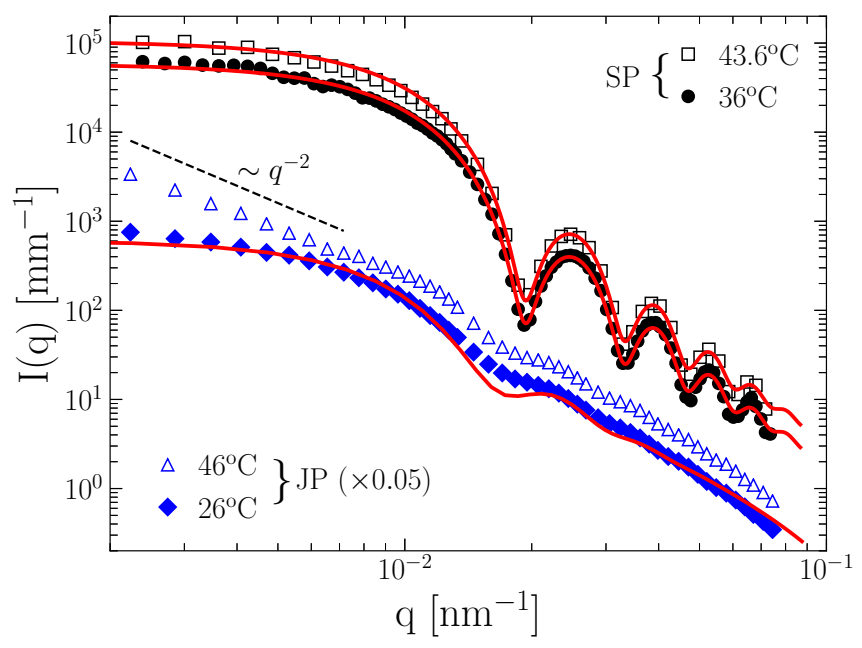

FIG. 4. Static USAXS profiles for SP and JP samples well below $T_{c}$ and their corresponding evolution above $T_{c}$. Red lines display modeling in terms of the scattering functions (form factor) of polydisperse homogeneous spheres $\left(R_{S}=231 \mathrm{~nm}\right.$ and $\left.P D=0.054\right)$ for SP and polydisperse spheres with a hemispherical cap $\left(R_{S}=200\right.$ $\mathrm{nm}, P D=0.15$ and $d_{\mathrm{Ni}} \approx 40 \mathrm{~nm}$ ) for JP. Scattering profiles of JP have been multiplied by a factor 0.05 for clarity.
USAXS profiles for SP and JP below and above $T_{c}$. The scattering profiles of SP sample are adequately described by noninteracting polydisperse sphere scattering function (red lines) [17] for the colloid concentrations used in this study (volume fraction $\sim 0.01$ ), i.e., the structure factor of interactions, $S(q) \approx 1$. Here the $\mathrm{T}$ jump bypassed the aggregation region of SP and the particles are repulsively stabilized at both temperatures. The main difference between the scattering profiles of SP below $T_{a}$ and above $T_{c}$ is that the concentration is roughly twice larger in the 3MP-rich phase since all particles have migrated to that phase above $T_{c}$ (the x-ray beam passed through the upper part of the cell where the 3MP-rich phase was formed). The scattering profile of the JP sample below $T_{a}$ can be described by a model incorporating the hemispherical nickel cap on to polydisperse silica spheres $[20,44]$. The mean radius of silica core obtained is smaller than the initial particles $\left(R_{S}=200 \mathrm{~nm}\right)$ with $P D \simeq 0.15$ and the thickness of the nickel cap is about $40 \mathrm{~nm}$. This suggests that the size distribution of particles changed in the processes of nickel plating and subsequent separation. The first minima in the data is more smeared than the model, which is attributed to some inhomogeneity of nickel caps and unseparated particle clusters (as suggested by slight upturn at low $q$ ). The polydispersity in the cap thickness is not included in the model. The thickness of the nickel cap is mainly determined by the sharper fall in the intensity at higher $q$ values and the discrepancy at the first minimum does not change the thickness value significantly. In the two-phase region, JP formed clusters as indicated by a sharp low- $q$ upturn in the scattering profile with a slope of -2 . In these measurements, the $\mathrm{x}$-ray beam traversed close to the position of the liquid-liquid interface. The $q^{-2}$ behavior may imply flat assembly of particles rather than mass fractal aggregates [20].

\section{Investigation of the dynamics}

In this section, the main results obtained from XPCS measurements are presented. The colloid dynamics in the one-phase region and two representative cases of the time evolution upon $\mathrm{T}$ jumps into the two-phase region of the solvent are described. Additional data are provided in Ref. [42].

\section{Colloid dynamics in the one-phase region}

For these studies the samples were heated stepwise to $40{ }^{\circ} \mathrm{C}$ and at each temperature XPCS measurements were performed to verify the stability of the suspension. Figure 5 shows the typical $g_{2}(q, t)$ functions for SP (a) and JP (b) systems well below $T_{c}$. As expected the decay of $g_{2}(q, t)$ is well described by a single exponential function, Eq. (2), and the corresponding relaxation rates are presented in the insets. The $q$ dependence of $\Gamma(q)$ is in agreement with passive Brownian particles, i.e., $\Gamma(q)=D_{0} q^{2}$. The derived $D_{0}$ value is lower for JP than the SP which can be attributed to the thicker nickel layer as well as possible dimers and larger clusters present in the suspension. Nevertheless, the functional form has not deviated from a single exponential decay. The $D_{0}$ value of SP system is consistent with previous work [17]. The corresponding $R_{H}$ is about $245 \mathrm{~nm}$ using the viscosity value of $1.6 \mathrm{mPa}$ for the critical solvent mixture [45]. 

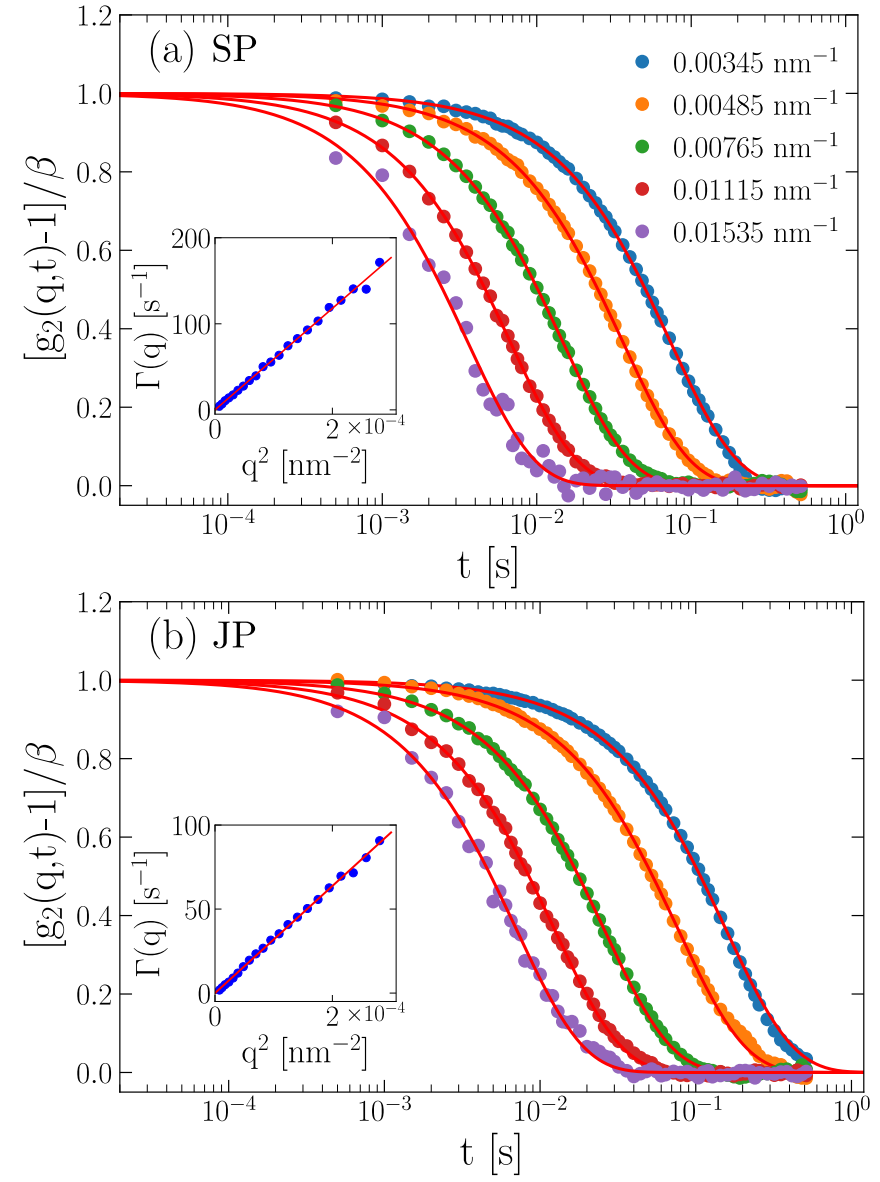

FIG. 5. Typical $g_{2}(q, t)$ functions for (a) SP and (b) JP depicting the normal diffusive dynamics before the $\mathrm{T}$ jump at $40{ }^{\circ} \mathrm{C}$. The red lines are fit curves using Eq. (2) with $\beta \simeq 0.32$. Insets present corresponding relaxation rates vs $q^{2}$. $D_{0}$ values $0.59 \mu \mathrm{m}^{2} / \mathrm{s}$ (SP) and $0.32 \mu \mathrm{m}^{2} / \mathrm{s}(\mathrm{JP})$ are obtained from the slope.

\section{Colloid dynamics upon T jumps into the two-phase region}

In these experiments, the sample was rapidly heated from $40{ }^{\circ} \mathrm{C}$ to the desired temperature above $T_{c}$ at the rate of $0.33 \mathrm{~K} / \mathrm{s}$, bypassing the aggregation region. The observed evolution of the dynamics is not sensitive to the starting temperature as long as it is below $T_{a}$. Typically the temperature equilibration is achieved in about $20 \mathrm{~s}$. But for the kinetics of phase separation, the time $t_{\text {kin }}$ started from 0 when the heating stage reached the final temperature. At this condition the phase separation of the solvent is expected to take place via the spinodal mechanism. Figures 6(a) and 6(b) display the typical evolution of dynamics upon a $\mathrm{T}$ jump of $\Delta T=1.3 \mathrm{~K}$ for SP and JP systems, respectively. Evidently, the dynamics has significantly enhanced and the azimuthally averaged $g_{2}(q, t)$ deviated from a simple exponential relaxation behavior. The leading contribution to the enhanced dynamics comes from the advection of particles upon phase separation of the solvent. The characteristic oscillations in the $g_{2}(q, t)$ functions indicate nearly constant $\delta v$ dominates the dynamics. Therefore Eq. (8) provided the best description of the data, which is also similar to that was found in the previous study [20]. However, in this case the initial decay of $g_{2}(q, t)$ is resolved by an order of magnitude better, which in turn permitted a direction
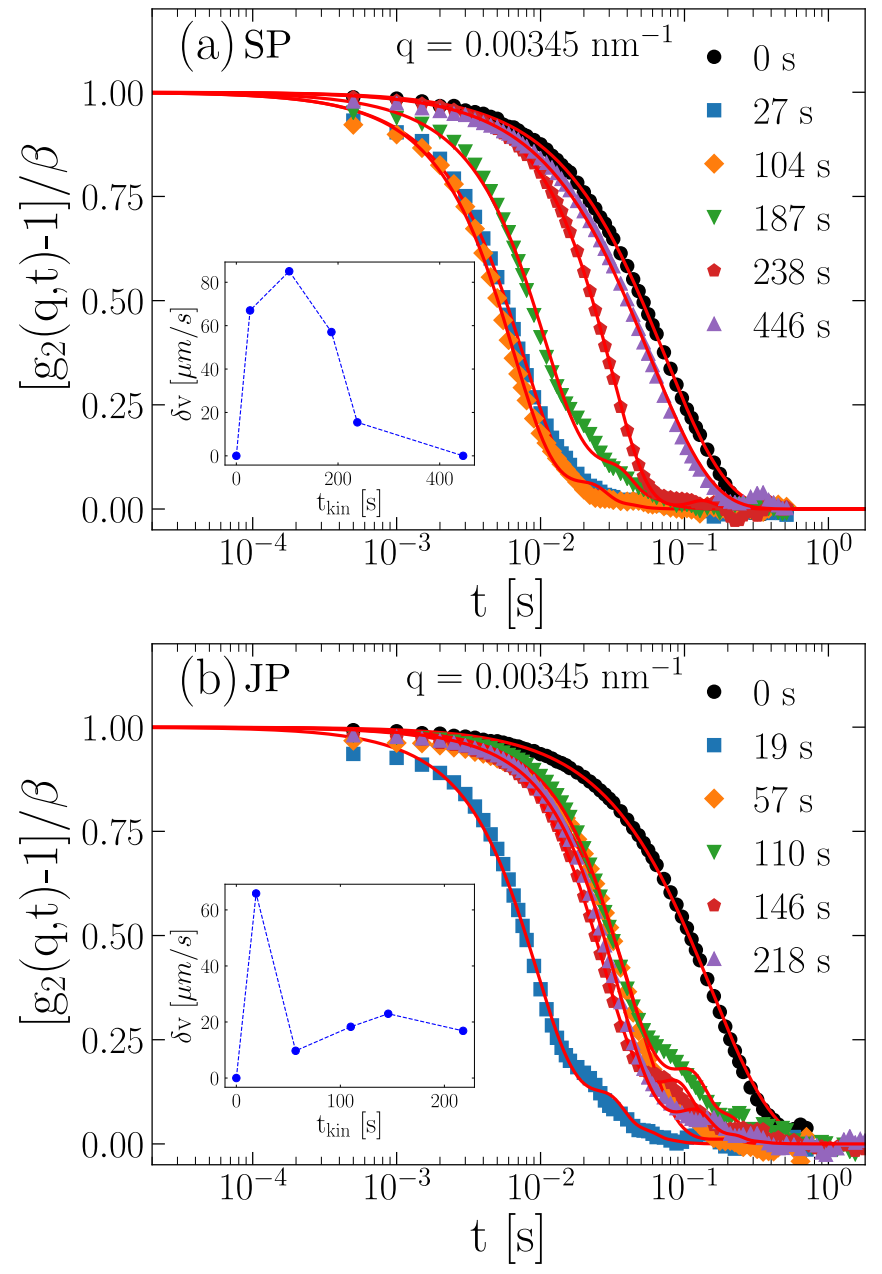

FIG. 6. Evolution of the azimuthally averaged $g_{2}(q, t)$ functions at $q=0.00345 \mathrm{~nm}^{-1}$ after a $\mathrm{T}$ jump of $\Delta T=1.3 \mathrm{~K}$ for (a) SP and (b) JP systems. The insets depict the time evolution of the velocity fluctuations, $\delta v$. The red lines are fit curves using Eq. (8) and small deviations at longer times are attributed to the anisotropy of the advective dynamics.

dependent analysis. The insets in Fig. 6 show the resulting $\delta v$ as a function of $t_{\text {kin. }}$. It is evident that $\delta v$ increases rapidly and then decays upon the progression of phase separation in the case of SP system while for JP sample the decay of $\delta v$ is even earlier. This difference is attributed to the accumulation of JP near the interfaces and associated clustering effect [20], while SP follow the 3MP-rich phase.

A better insight into this out-of-equilibrium dynamics can be obtained by the direction dependent analysis of $g_{2}(q, t)$. Figures 7 and 8 display representative $g_{2}(q, t)$ functions along the horizontal, $g_{2}\left(q_{\perp}, t\right)$, and vertical, $g_{2}\left(q_{\|}, t\right)$, directions for SP and JP systems. Additional sets of data can be found in Ref. [42]. It is clear that the dynamics changed immediately upon the sample reaching the set temperature. Both $g_{2}\left(q_{\perp}, t\right)$ and $g_{2}\left(q_{\|}, t\right)$ are well-described by Eq. (8). In the one-phase region, the correlation length of concentration fluctuations exceeds the particle size near $T_{c}$ [23]. When a critical binary fluid mixture undergoes phase separation, the fluid velocity fluctuations are expected to follow the concentration fluctuations in the mode-coupling analysis of the spinodal dynamics 


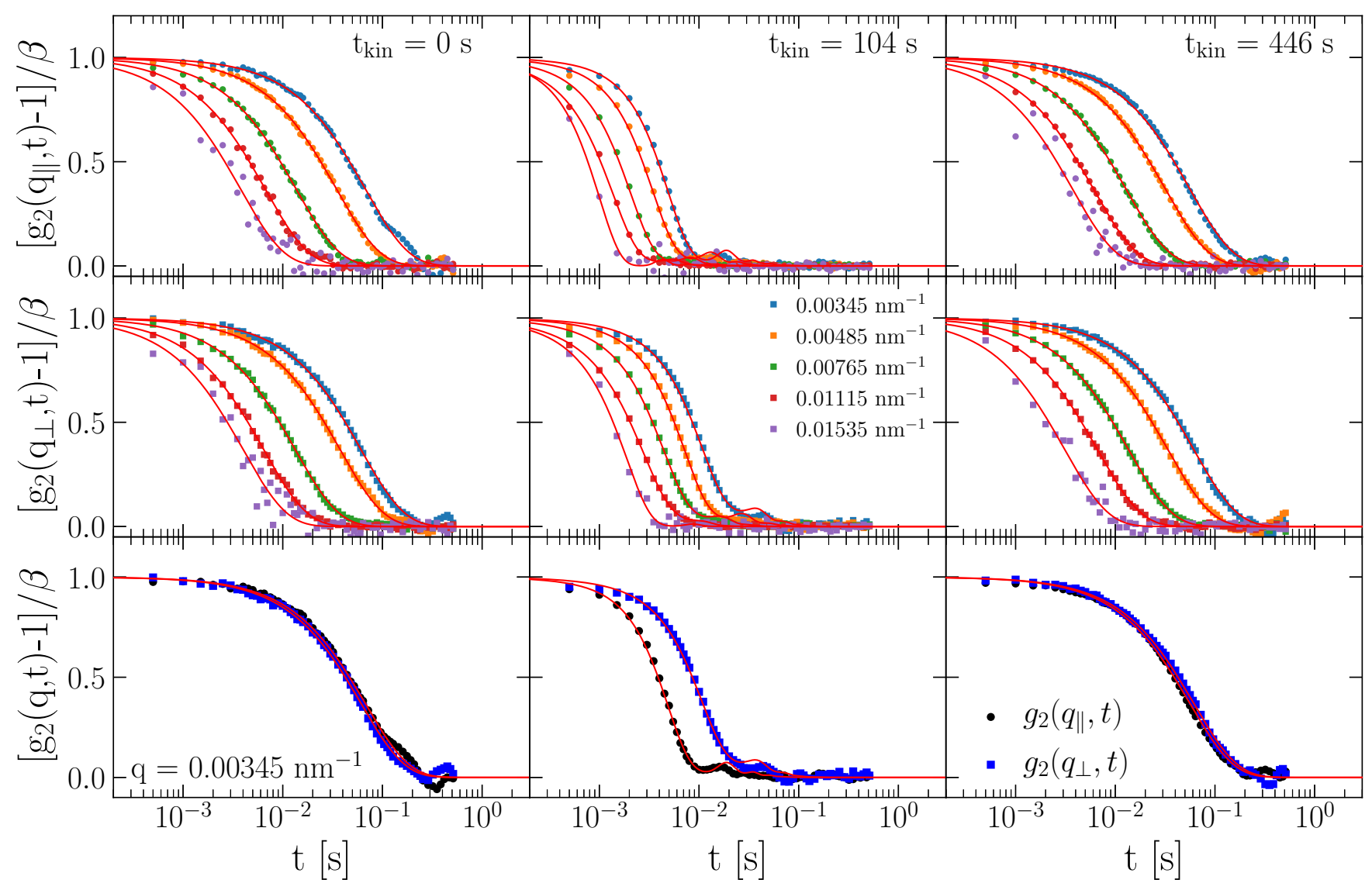

FIG. 7. Direction dependent analysis of $g_{2}(q, t)$ functions for SP system: the top row presents $g_{2}\left(q_{\|}, t\right)$ in the vertical direction at three different times during phase separation and the middle row displays the corresponding $g_{2}\left(q_{\perp}, t\right)$ for the horizontal direction. For better comparison between $g_{2}\left(q_{\|}, t\right)$ and $g_{2}\left(q_{\perp}, t\right)$, they are plotted together for $q=0.00345 \mathrm{~nm}^{-1}$ in the bottom row. Red lines are fits to Eq. (8).

$[30,46]$. Therefore, the initial velocity fluctuations $\left(t_{\text {kin }}<\right.$ $30 \mathrm{~s}$ ) are likely induced by the large concentration fluctuations in the solvent upon $\mathrm{T}$ jump into the unstable region [26,28]. At later times, the faster advective motion of the particles along the vertical direction is likely due to gravity-driven hydrodynamic flow when the domain sizes become larger than the typical capillary length, $l_{c}$ [27,29,31]. Where $l_{c} \simeq$ $\sqrt{\sigma / \Delta \rho g}, \sigma, \Delta \rho$, and $g$ are the interfacial tension, density difference and acceleration due to gravity, respectively. An additional complication is that in the two-phase region just above $T_{c}$, the solvent mixture is in a complete wetting regime [11] that in the presence of SP, the 3MP-rich phase wets the walls of the container [10]. Therefore the faster dynamics along the vertical direction compared to that in the horizontal direction, may also be augmented by the dynamic coupling between wetting and phase separation [31]. Irrespective of the mechanism, the faster dynamics along $q_{\|}$signifies the net advection of particles along the vertical direction to the preferred phase as schematically depicted in Figs. 2(b) and 2(c). The conventional sedimentation of particles to the bottom of capillary under the influence of gravity could also lead to a similar anisotropic advective dynamics [41]. However, the effect of sedimentation is excluded from the concentration profile observed by the USAXS intensities after each XPCS measurement and by direct observation of the sample (few particles were found at the bottom region of the capillary).
Since, both SP and JP systems display a similar behavior for horizontal and vertical dynamics, the enhancement of dynamics is primarily contributed by the concentration fluctuations and hydrodynamic flow associated with the late stage of the spinodal phase separation [27,31].

Figure 9 presents the main fit parameters $D_{\text {eff }}, \delta v$, and $\alpha$ from the direction dependent analysis. The fluctuations in the parameter values suggest intermittent behavior, which could be smoothed out by longer averaging of the $g_{2}(q, t)$ measurement. The faster dynamics of colloids after the $\mathrm{T}$ jump is also associated with a larger $D_{\text {eff }}$ that is consistent with previous results [17]. The earlier slowing down of the advective dynamics in the case of JP is clearly visible in the fit parameters for $D_{\text {eff }}$ and $\delta v$, which is attributed to dynamic clustering effect. The $D_{\text {eff }}$ presented in Fig. 9 are derived assuming $q^{2}$ dependence of $\Gamma_{\text {eff }}(q)$. By analogy with active Brownian particles, the $D_{\text {eff }}$ can be related to the Péclet number $(P e)$ of directed motion, $D_{\text {eff }}=D_{0}(1+$ $\left.2 / 9 P e^{2}\right)[6,47]$. Where $P e$ is given by the ratio of diffusive and ballistic times, $P e=2 R_{S} \times v / D_{0}$. This enables to deduce independently $v$ from the derived $D_{\text {eff }}$ values. The maximum value of $D_{\text {eff }}$ presented in Fig. 9 corresponds to $v \simeq$ $10 \mu \mathrm{m} / \mathrm{s}$ which is consistent with the time scale of the appearance of clear macroscopic liquid-liquid interface. In addition, this value of $v$ justifies the assumption $\left|g_{1, T}(q, t)\right|^{2} \approx 1$ in Eq. (5). 


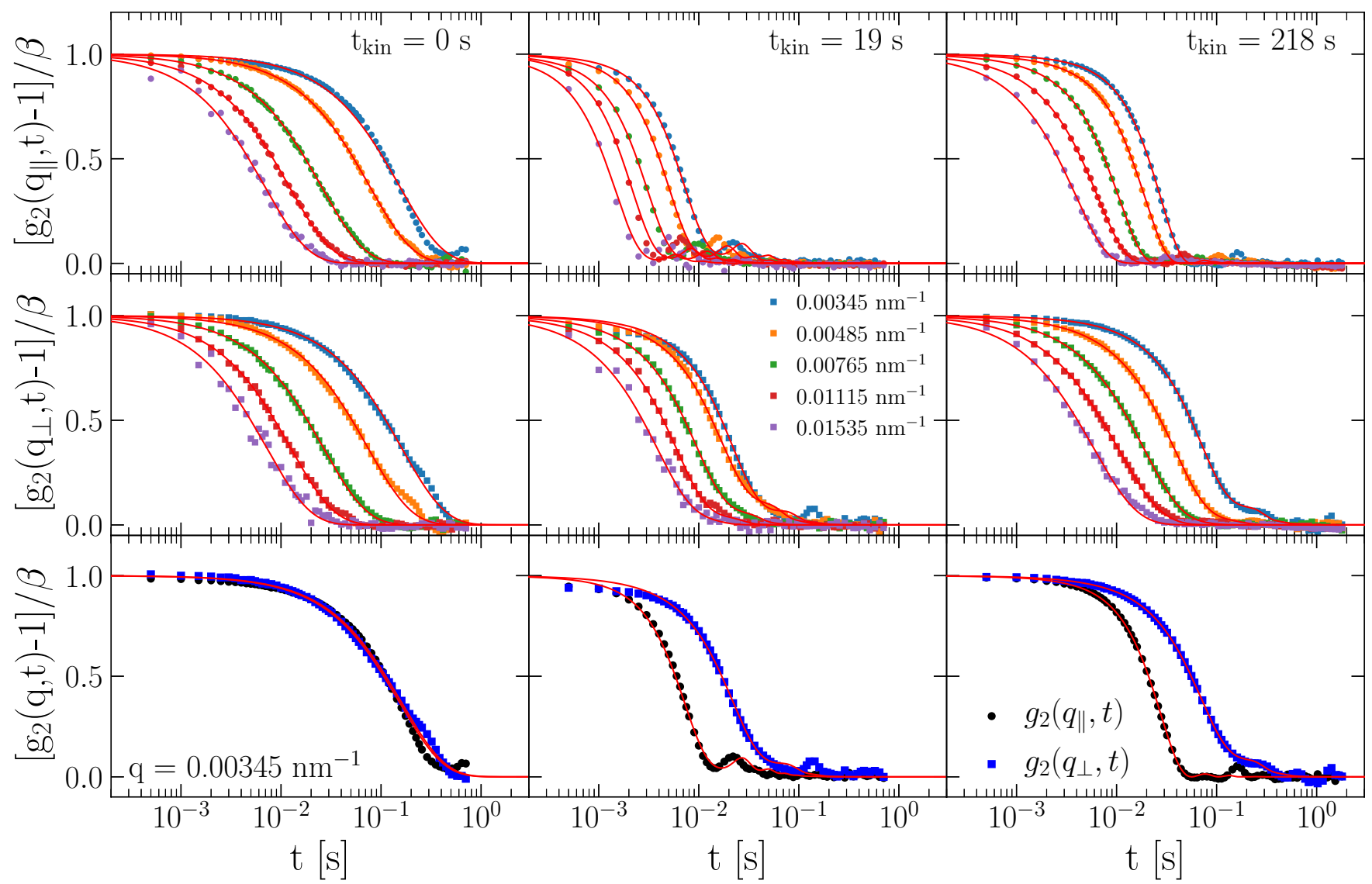

FIG. 8. Direction dependent analysis of $g_{2}(q, t)$ functions for JP system: the top row presents $g_{2}\left(q_{\|}, t\right)$ in the vertical direction at three different times during phase separation and the middle row displays the corresponding $g_{2}\left(q_{\perp}, t\right)$ for the horizontal direction. For better comparison between $g_{2}\left(q_{\|}, t\right)$ and $g_{2}\left(q_{\perp}, t\right)$, they are plotted together for $q=0.00345 \mathrm{~nm}^{-1}$ in the bottom row. Red lines are fits to Eq. (8).
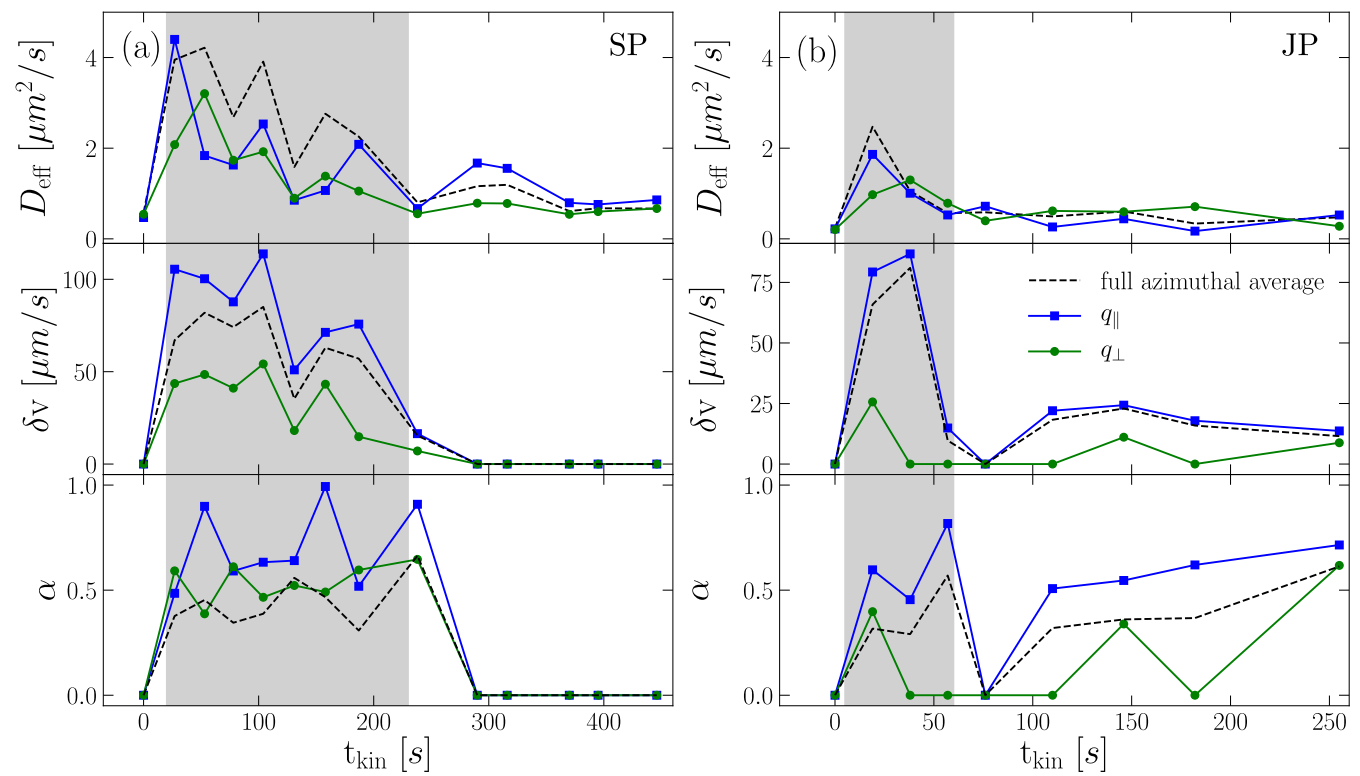

FIG. 9. Fit parameters from the direction dependent analysis presented in Figs. 7 and 8 for (a) SP and (b) JP systems, respectively. The parameters increase rapidly upon the sample reaching the set temperature. In JP system, the parameters decay abruptly due to clustering effect and the longer time behavior may also be influenced by the sedimentation of some clusters. Shaded areas are guide to the eye showing the enhanced dynamics. 

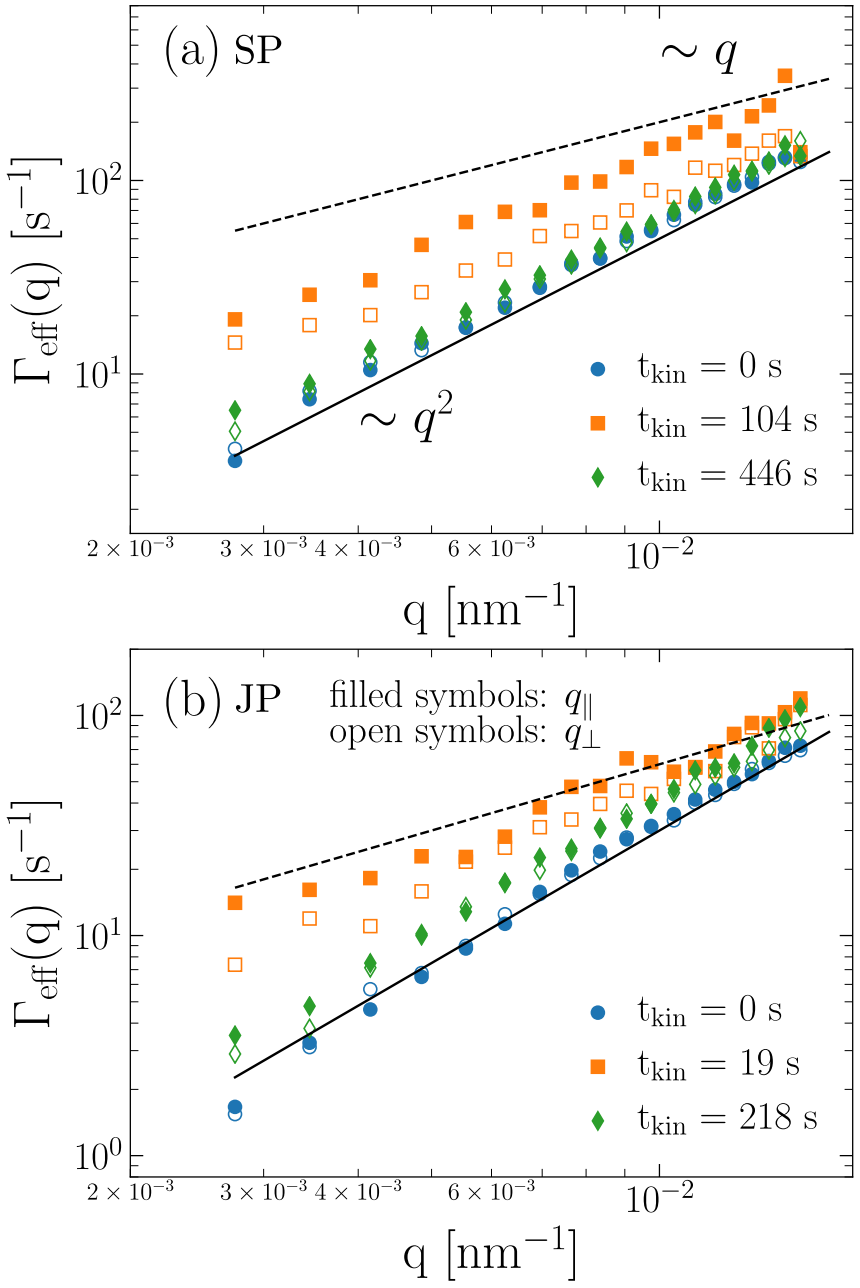

FIG. 10. Effective relaxation rates, $\Gamma_{\text {eff }}$ vs $q$ obtained from the analysis shown in Figs. 7 and 8 for (a) SP and (b) JP systems, respectively at representative $t_{\text {kin }}$. The $q$ dependence changed from quadratic to a smaller exponent that may signify a superdiffusive behavior.

Further difference from passive Brownian particles is evident in the $q$ dependence of $\Gamma_{\text {eff }}$ at different $t_{\text {kin }}$ shown in Fig. 10. The faster decays are characterized by a weaker $q$ dependence of $\Gamma_{\text {eff }}$ than $q^{2}$ behavior in Fig. 5. This suggests that the diffusion of particles has become anomalous with an enhanced $D_{\text {eff. }}$. Furthermore, $\Gamma_{\text {eff }} \sim q^{\mu}$ with $\mu<2$ in both systems signify an anomalous diffusion analogous to superdiffusion [48]. This superdiffusive process may involve Lévy flight like steps [49], while particles transport through the bicontinuous domains of the phase separated solvent following spinodal decomposition [31]. This means that the particle diffusion is strongly coupled with the concentration fluctuations in the solvent. For symmetric active Brownian particles (without net propulsion), a superdiffusive regime is expected between inertial (ballistic motion without dissipation) and diffusive ranges [22]. In the case of asymmetric active particles (with net propulsion), propulsive and anomalous modes coexist over a certain time range below the rotational diffusion time [22]. In summary, both SP and JP systems display certain hallmarks

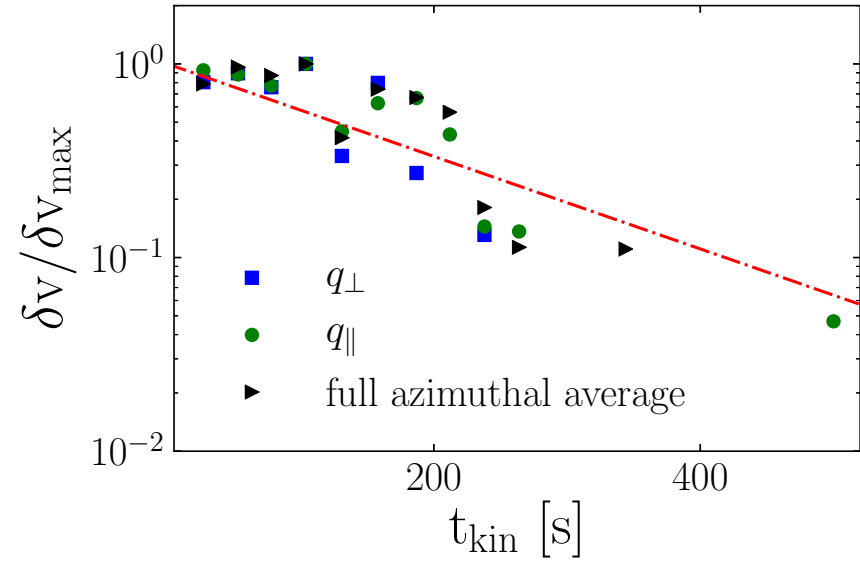

FIG. 11. The decay of velocity fluctuations, $\delta v$ normalized by its maximum value for SP system. The spread is roughly exponential, which is similar to the behavior in colloidal sedimentation [41].

of active colloids when the solvent mixture undergoes phase separation.

Indeed, a weaker $q$ dependence may also arise from the remnant coupling with the advective term. In other words, the advective term is not fully accounted by a uniform distribution of $\delta v$. However, the characteristic features of $g_{2}\left(q_{\|}, t\right)$ and $g_{2}\left(q_{\perp}, t\right)$ functions justify the assumption of constant $\delta v$. In addition, both SP and JP systems show a similar trend in the $q$ dependence of $\Gamma_{\text {eff }}$. The anomalous $q$ dependence of $\Gamma_{\text {eff }}$ could not be resolved in previous studies $[17,20]$, because of the limited time range of the initial decay of the measured $g_{2}(q, t)$ functions. Similar behavior was observed for deeper jumps upto $\Delta T=5 \mathrm{~K}$ except that the dynamics became faster and the phase separation was completed in a shorter time. For deeper T jumps JPs tend to form larger clusters, that resulted in a slowly decaying tail in the $g_{2}(q, t)$ function, as already observed in the previous study [20].

At the late stage of phase separation, the average domain size $\left(a_{\mathrm{d}}\right)$ is expected to follow a power-law, $a_{\mathrm{d}} \sim t_{\text {kin }}^{n}$, with the exponent, $n \simeq 1-1.5$ depending on the specific coarsening mechanism $[29,31,50]$. Parameters $\delta v$ and $D_{\text {eff }}$ in Fig. 9 do not show a straightforward relationship with $a_{\mathrm{d}}$. This demonstrates that the induced dynamics is controlled by the composition fluctuations between different domains within the scattering volume than the domain size. The dynamics reverts to normal diffusive behavior when the solvent composition fluctuations and hydrodynamic flow subside prior to the appearance of the macroscopic liquid-liquid interface. In the case of SP system, the decay behavior of $\delta v$ is roughly exponential as shown in Fig. 11, which is similar to that found for sedimentation [41]. This similarity of late stage of phase separation with sedimentation has already been suggested [27]. In JP system, the clustering effect dominates the dynamics at later stages that makes the decay of $\delta v$ more abrupt and much earlier than the completion of domain growth.

\section{Evolution of dynamics for smaller $T$ jumps into the two-phase region}

As the final temperature becomes closer to $T_{c}$, the interfacial tension almost vanishes [11], which makes it 

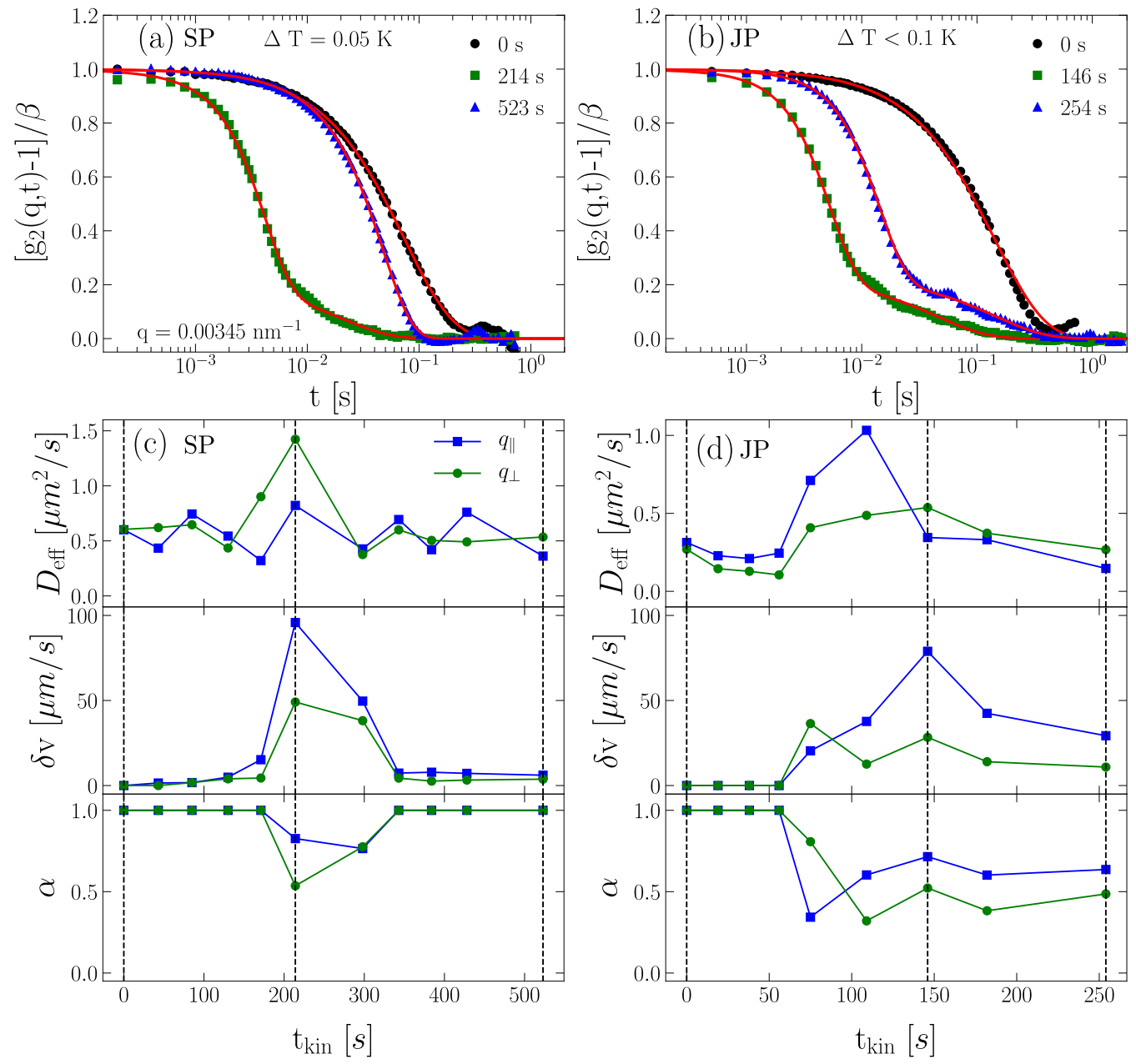

FIG. 12. Representative azimuthally averaged $g_{2}(q, t)$ functions for small $\mathrm{T}$ jumps: (a) $\Delta T \approx 0.05 \mathrm{~K}$ for SP and (b) $\Delta T \leqslant 0.1 \mathrm{~K}$ for JP systems. The fit curves correspond to Eq. (9). The lower panels (c) and (d) show the corresponding fit parameters from direction dependent analysis shown in Figs. S6 and S7 for SP and JP systems, respectively. Dashed vertical lines represent $t_{\text {kin }}$ values for azimuthally averaged $g_{2}(q, t)$ functions shown in the upper panel. Notice that the enhanced dynamics of JP system persists for longer times.

insufficient to support the accumulation of particles at the liquid-liquid interface in the JP system. As a result, the particle layers collapsed to the bottom of the capillary. This sedimentation effect prevented measurement of the dynamics for longer times. Since, the concentration of 3MP is critical, the phase separation is still expected to take place via spinodal decomposition.

Figure 12 presents the observed behavior of SP and JP samples for $\mathrm{T}$ jumps just above $T_{c}(\Delta T \lesssim 0.1 \mathrm{~K})$. Qualitatively, there is a difference in the functional form of the decay of $g_{2}(q, t)$. While for JP the $g_{2}(q, t)$ functions display the features of Eq. (8) as in the case of larger $\mathrm{T}$ jumps, SP dynamics can be described by Eq. (6) at least at shorter times. In order to make the comparison straightforward, $g_{2}(q, t)$ functions in both systems were fitted to Eq. (9). This corresponds to the velocity fluctuations following a Gaussian distribution instead of a constant profile. This observation is consistent with the assumption of Gaussian distribution of concentration fluctuations in the mode-coupling analysis of spinodal decomposition [46]. Furthermore, it is similar to the velocity fluctuations in turbulent convection [51] and this analogy between the phase separation and fully developed turbulence has already been suggested [30]. Figure 12 (bottom panel) shows the corresponding fit parameters $D_{\text {eff }}, \delta v$, and $\alpha$ for SP and JP systems. Since Eq. (9) has the same $q$ dependence for both advective and diffusive terms, it is difficult to completely decouple the $q$ dependence

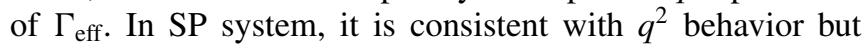
JP system shows a clear trend to a weaker $q$ dependence of $\Gamma_{\text {eff }}$ as presented in Fig. 13(b). The full direction-dependent analyses are presented in Figs. S6 and S7 [42]. Once again, the time evolution of parameters does not display a direct correspondence with the expected domain growth dynamics, $a_{\mathrm{d}} \sim t_{\mathrm{kin}}^{n}$.

In SP system, after a short burst $D_{\text {eff }}$ remained at the expected range of $D_{0}$ and velocity fluctuations displayed smaller magnitude and shorter duration as compared to larger T jumps. On the other hand, the superdiffusive-like motions in JP system $\left(\Gamma_{\text {eff }} \sim q^{\mu}\right.$ with $\left.\mu<2\right)$ did not decay rapidly unlike in the case of larger $\mathrm{T}$ jumps. This may be an indication of the coexistence of propulsion and anomalous diffusion as predicted for asymmetric active Brownian particles [22]. Figure S8 shows the behavior of $g_{2}(q, t)$ for JP system when the jump temperature was below $T_{c}$ [42]. There was no significant 

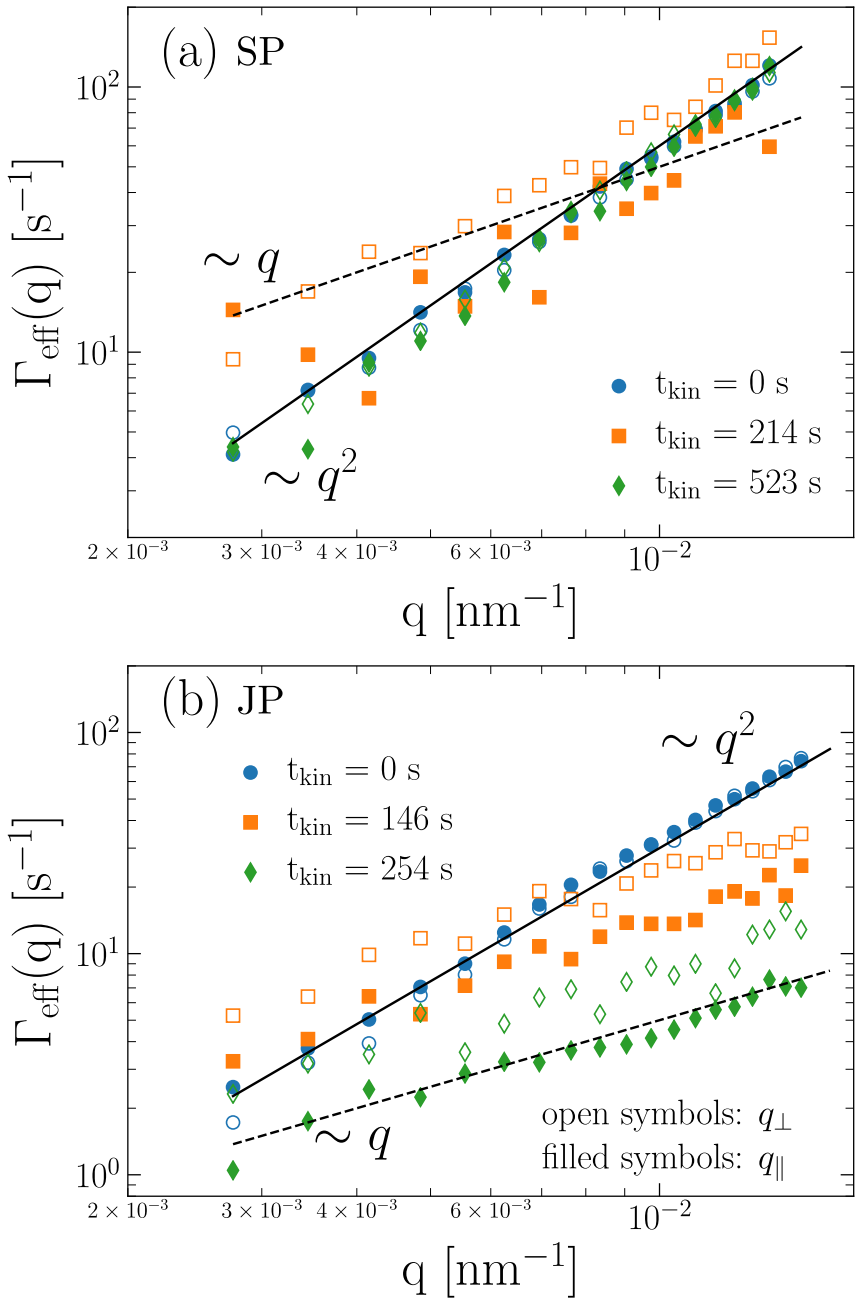

FIG. 13. Effective relaxation rates, $\Gamma_{\text {eff }}$ vs $q$ obtained from the direction dependent analysis of the data in Fig. 12 and presented in Figs. S6 and S7 on a log-log representation for (a) SP and (b) JP systems. The JP system shows a trend towards linear $q$ dependence.

change in the dynamics in that case, clearly demonstrating that the induced fast dynamics observed in the previous cases are intimately related to phase separation of the solvent. With SP sample, a similar T jump protocol cannot be performed because particles reversibly aggregate below $T_{c}$. Moreover, near $T_{c}$ of the binary mixture, colloidal interactions are further complicated by critical Casimir interactions [12] and dynamic coupling between wetting and phase separation mentioned above [31].

\section{SUMMARY AND CONCLUSION}

The results presented in the previous sections illustrate the peculiar dynamic behavior of colloids when the solvent is undergoing a liquid-liquid phase separation. The combined effects of phase separation and wetting result in migration of colloids to specific regions depending on the surface specificity (reduction of surface energy) of the particles. In the case of SP, particles migrate to the 3MP-rich phase while JP accumulate near the liquid-liquid interface. The associated phoretic dynamics is determined by the concen- tration fluctuations and gravity induced hydrodynamic flow for larger $\mathrm{T}$ jumps into the two-phase region. Large velocity fluctuations dominate the observed dynamics, which is reminiscent of early stage of sedimentation [41]. In addition, the diffusive part of the dynamics becomes anomalous with a superdiffusive-like $q$ dependence [48]. The JP system also shows similar dynamics at the early stage for larger T jumps, though the phase behavior is significantly different. The particle clustering effect interrupts the enhanced dynamics induced by the phase separation of the solvent. At longer times, Janus particles cluster into different configurations and partition in the coexisting phases. Nevertheless, it can be concluded that in both systems, the observed phoretic dynamics is intimately related to the concentration fluctuations during the spinodal phase separation of the solvent mixture without a direct correspondence with the expected domain growth kinetics. The link between the anomalous diffusion and the concentration fluctuations needs to be scrutinized more quantitatively by computer simulations.

The direction dependent analysis showed that the dynamics is not completely isotropic during the phase separation process though the functional form of $g_{2}(q, t)$ is not altered significantly. For smaller $\mathrm{T}$ jumps, the phase separation kinetics appeared to affect the colloid dynamics only at the early stage. At the late stage of phase separation, the colloid dynamics reverted to more diffusive-like behavior in the SP system. In other words, the hydrodynamic flow was not sufficient to modify the colloid dynamics dramatically (low $P e$ value). In the JP system, the enhanced dynamics persists for longer times, which is likely the most relevant condition for activity of the asymmetric particles upon self-heating that leads to local phase separation of the solvent [8]. The overall evolution of the dynamics closely resembles the observations in previous studies $[17,20]$ involving nucleation and growth. This may be attributed to the similarities in the concentration gradients within the coarsening patterns at the late stages of phase separation in both cases. Main differences are in the extent of velocity fluctuations, the anisotropy and the faster onset of enhanced dynamics in the spinodal case.

USAXS and USA-XPCS enabled probing the change in colloid microstructure and dynamics in turbid systems when the solvent is undergoing spinodal phase separation. The measured $g_{2}(q, t)$ functions can be decomposed to advective and diffusive components of particle dynamics. Therefore, these scattering methods are suitable for the investigation of concentrated active colloids. In particular, the relevant $q$ and time scales involved in active dynamics can be accessed in 3D systems. This could be an alternative approach for probing active Brownian dynamics upon turning on the activity by light [52] and derive quantitative information on their stochastic behavior in 3D. It will be also interesting to study the organization and dynamics of active Janus particles at the liquid-liquid interface using surface sensitive grazing incidence SAXS and XPCS methods.

\section{ACKNOWLEDGMENTS}

The ID02 beamline staff, Y. Chushkin (ESRF) and I. Snigireva (Microimaging Lab, ESRF) are thanked for technical support, PyXPCS software package and SEM 
characterization, respectively. The ESRF is acknowledged for the provision of synchrotron beam time and financial support. The work benefited from the Electron Microscopy facility at the Institut de Biologie Structurale (IBS) as part of Grenoble Instruct-ERIC Center (ISBG : UMS 3518) and D. Fenel is thanked for assistance.
[1] R. Kapral, Perspective: Nanomotors without moving parts that propel themselves in solution, J. Chem. Phys. 138, 020901 (2013).

[2] J. Zhang, E. Luijten, B. A. Grzybowski, and S. Granick, Active colloids with collective mobility status and research opportunities, Chem. Soc. Rev. 46, 5551 (2017).

[3] P. Illien, R. Golestanian, and A. Sen, 'fuelled' motion: phoretic motility and collective behavior of active colloids, Chem. Soc. Rev. 46, 5508 (2017).

[4] M. C. Marchetti, J.-F. Joanny, S. Ramaswamy, T. B. Liverpool, J. Prost, M. Rao, and R. A. Simha, Hydrodynamics of soft active matter, Rev. Mod. Phys. 85, 1143 (2013).

[5] C. Bechinger, R. Di Leonardo, H. Löwen, C. Reichhardt, G. Volpe, and G. Volpe, Active particles in complex and crowded environments, Rev. Mod. Phys. 88, 045006 (2016).

[6] A. Zöttl and H. Stark, Emergent behavior in active colloids, J. Phys.: Condens. Matter 28, 253001 (2016).

[7] J. L. Moran and J. D. Posner, Phoretic self-propulsion, Annu. Rev. Fluid Mech. 49, 511 (2017).

[8] I. Buttinoni, G. Volpe, F. Kümmel, G. Volpe, and C. Bechinger, Active brownian motion tunable by light, J. Phys.: Condens. Matter 24, 284129 (2012).

[9] I. Buttinoni, J. Bialké, F. Kümmel, H. Löwen, C. Bechinger, and T. Speck, Dynamical Clustering and Phase Separation in Suspensions of Self-Propelled Colloidal Particles, Phys. Rev. Lett. 110, 238301 (2013).

[10] D. Beysens and T. Narayanan, Wetting-induced aggregation of colloids, J. Stat. Phys. 95, 997 (1999).

[11] B. M. Law, Wetting, adsorption and surface critical phenomena, Prog. Surf. Sci. 66, 159 (2001).

[12] A. Maciołek and S. Dietrich, Collective behavior of colloids due to critical casimir interactions, Rev. Mod. Phys. 90, 045001 (2018).

[13] B. M. Law, J.-M. Petit, and D. Beysens, Adsorption-induced reversible colloidal aggregation, Phys. Rev. E 57, 5782 (1998).

[14] A. Würger, Self-Diffusiophoresis of Janus Particles in NearCritical Mixtures, Phys. Rev. Lett. 115, 188304 (2015).

[15] S. Samin and R. van Roij, Self-Propulsion Mechanism of Active Janus Particles in Near-Critical Binary Mixtures, Phys. Rev. Lett. 115, 188305 (2015).

[16] T. Araki and A. Maciołek, Illumination-induced motion of a janus nanoparticle in binary solvents, Soft Matter 15, 5243 (2019).

[17] R. Dattani, E. F. Semeraro, and T. Narayanan, Phoretic motion of colloids in a phase separating medium, Soft Matter 13, 2817 (2017).

[18] C. Yu, J. Zhang, and S. Granick, Selective janus particle assembly at tipping points of thermally-switched wetting, Angew. Chem., Int. Ed. 53, 4364 (2014).

[19] T. A. Nguyen, A. Newton, S. J. Veen, D. J. Kraft, P. G. Bolhuis, and P. Schall, Switching colloidal superstructures by critical casimir forces, Adv. Mater. 29, 1700819 (2017).
[20] E. F. Semeraro, R. Dattani, and T. Narayanan, Microstructure and dynamics of janus particles in a phase separating medium, J. Chem. Phys. 148, 014904 (2018).

[21] R. L. Leheny, M. C. Rogers, K. Chen, S. Narayanan, and J. L. Harden, Rheo-XPCS, Curr. Opin. Colloid Interface Sci. 20, 261 (2015).

[22] R. Golestanian, Anomalous Diffusion of Symmetric and Asymmetric Active Colloids, Phys. Rev. Lett. 102, 188305 (2009).

[23] T. Narayanan and A. Kumar, Reentrant phase transitions in multicomponent liquid mixtures, Phys. Rep. 249, 135 (1994).

[24] K. Binder and P. Fratzl, Spinodal Decomposition, in Phase Transformations in Materials (Wiley VCH, Weinheim, 2001), pp. 409-480.

[25] N.-C. Wong and C. M. Knobler, Light scattering studies of phase separation in isobutyric acid + water mixtures, J. Chem. Phys. 69, 725 (1978).

[26] Y. C. Chou and W. I. Goldburg, Angular distribution of light scattered from critically quenched liquid mixtures, Phys. Rev. A 23, 858 (1981).

[27] C.-K. Chan and W. I. Goldburg, Late-Stage Phase Separation and Hydrodynamic Flow in a Binary Liquid Mixture, Phys. Rev. Lett. 58, 674 (1987).

[28] P. Guenoun, R. Gastaud, F. Perrot, and D. Beysens, Spinodal decomposition patterns in an isodensity critical binary fluid: Direct-visualization and light-scattering analyses, Phys. Rev. A 36, 4876 (1987).

[29] E. D. Siggia, Late stages of spinodal decomposition in binary mixtures, Phys. Rev. A 20, 595 (1979).

[30] K. Kawasaki and T. Ohta, Kinetics of fluctuations for systems undergoing phase transitions-interfacial approach, Phys. A 118, 175 (1983).

[31] H. Tanaka, Interplay between wetting and phase separation in binary fluid mixtures: roles of hydrodynamics, J. Phys.: Condens. Matter 13, 4637 (2001).

[32] T. Zinn, A. Homs, L. Sharpnack, G. Tinti, E. Fröjdh, P. Douissard, M. Kocsis, J. Möller, Y. Chushkin, and T. Narayanan, Ultra-small-angle $\mathrm{x}$-ray photon correlation spectroscopy using the eiger detector., J. Synchrotron Radiat. 25, 1753 (2018).

[33] T. Narayanan, M. Sztucki, P. V. Van, J. Léonardon, J. Gorini, L. Claustre, F. Sever, J. Morse, and P. Boesecke, A multipurpose instrument for time-resolved ultra-small-angle and coherent X-ray scattering., J. Appl. Crystallogr. 51, 1511 (2018).

[34] B. Berne and R. Pecora, Dynamic Light Scattering: With Applications to Chemistry, Biology, and Physics (Dover Publications Inc, Mineola, New York, 2000).

[35] P. H. P. Chang and S. S. Penner, Determinations of turbulent velocity fluctuations and mean particle radii in flames using scattered laser-power spectra, J. Quant. Spectrosc. Radiat. Transfer 25, 97 (1981).

[36] S. Busch, T. H. Jensen, Y. Chushkin, and A. Fluerasu, Dynamics in shear flow studied by x-ray photon correlation spectroscopy, Eur. Phys. J. E: Soft Matter Biol. Phys. 26, 55 (2008). 
[37] W. R. Burghardt, M. Sikorski, A. R. Sandy, and S. Narayanan, $\mathrm{X}$-ray photon correlation spectroscopy during homogenous shear flow, Phys. Rev. E 85, 021402 (2012).

[38] P. Tong, W. I. Goldburg, C.-K. Chan, and A. Sirivat, Turbulent transition by photon-correlation spectroscopy, Phys. Rev. A Gen. Phys. 37, 2125 (1988).

[39] R. Weber and G. Schweiger, Photon correlation spectroscopy on flowing polydisperse fluid-particle systems: theory, Appl. Opt. 37, 4039 (1998).

[40] H. K. Pak, W. I. Goldburg, and A. Sirivat, An experimental study of weak turbulence, Fluid Dynamics Research 8, 19 (1991).

[41] J. Möller and T. Narayanan, Velocity Fluctuations in Sedimenting Brownian Particles, Phys. Rev. Lett. 118, 198001 (2017).

[42] See Supplemental Material at http://link.aps.org/supplemental/ 10.1103/PhysRevResearch.2.033177 for additional XPCS data and analysis for different $\mathrm{T}$ jumps and schematic phase diagram of the SP system.

[43] M. Pu, H. Jiang, and Z. Hou, Reentrant phase separation behavior of active particles with anisotropic janus interaction, Soft Matter 13, 4112 (2017).

[44] H. Kaya, Scattering from cylinders with globular end-caps, J. Appl. Crystallogr. 37, 223 (2004).
[45] G. A. Larsen and C. M. Sorensen, Shear-Viscosity Behavior Near the Double Critical Point of the Mixture 3-methylpyridine, Water, and Heavy Water, Phys. Rev. Lett. 54, 343 (1985).

[46] K. Kawasaki and T. Ohta, Theory of early stage spinodal decomposition in fluids near the critical point. II, Prog. Theor. Phys. 59, 362 (1978).

[47] D. Loi, S. Mossa, and L. F. Cugliandolo, Effective temperature of active matter, Phys. Rev. E 77, 051111 (2008).

[48] J.-P. Bouchaud, Anomalous relaxation in complex systems: From stretched to compressed exponentials, in Anomalous Transport: Foundations and Applications (Wiley, VCH, Weinheim, 2008), Chap. 11, pp. 327-345.

[49] J.-P. Bouchaud and A. Georges, Anomalous diffusion in disordered media: statistical mechanisms, models and physical applications, Phys. Rep. 195, 127 (1990).

[50] A. Cumming, P. Wiltzius, F. S. Bates, and J. H. Rosedale, Lightscattering experiments on phase-separation dynamics in binary fluid mixtures, Phys. Rev. A 45, 885 (1992).

[51] Y. Shen, K.-Q. Xia, and P. Tong, Measured Local-Velocity Fluctuations in Turbulent Convection, Phys. Rev. Lett. 75, 437 (1995).

[52] S. Palagi, D. P. Singh, and P. Fischer, Light-controlled micromotors and soft microrobots, Adv. Opt. Mater. 7, 1900370 (2019). 